\title{
Risk perception of medicinal marijuana in medical students
}

\section{from northeast Mexico [version 1; peer review: 1 approved}

\section{with reservations, 1 not approved]}

\author{
Sandra Castillo-Guzmán1, Dionicio Palacios-Ríos', Teresa A. Nava-Obregón1， \\ Julio C. Arredondo-Mendoza1, Olga V. Alcalá-Alvarado1, Sofía A. Alonso-Bracho1, \\ Daniela A. Becerril-Gaitán1, Omar González-Santiago (iD) \\ ${ }_{1}^{1}$ Pain and Palliative Care Clinic, Anesthesiology Service, Hospital Universitario Dr. José E. González y Facultad de Medicina, \\ Universidad Autónoma de Nuevo León, Monterrey, Nuevo León, Mexico \\ 2Postgraduate in Pharmacy, Faculty of Chemical Science, Universidad Autónoma de Nuevo León, San Nicolas de Los Garza, Nuevo \\ León, Mexico
}

V1 First published: 04 Oct 2017, 6:1802

https://doi.org/10.12688/f1000research.12638.1

Latest published: 04 Oct 2017, 6:1802

https://doi.org/10.12688/f1000research.12638.1

\section{Abstract}

Background. Several studies have shown support from the public toward the use of medicinal marijuana. In this cross-sectional study, we assess the risk perception to medicinal marijuana in a sample of medical students.

Methods. To estimate risk perception, a visual scale that ranges from $0 \mathrm{~cm}$ (without risk) to $10 \mathrm{~cm}$ (totally risky) was used. Risk perception was expressed as the median of the $\mathrm{cm}$ marked over the scale. Differences among groups was tested with the Mann-Whitney and Kruskal-Wallis tests, as appropriate.

Results. 283 students participated in the study. Risk perception to medicinal marijuana was 4.22, paracetamol 1.56 and sedatives 5.0. A significant difference in risk perception was observed in those that self-reported to smoke and consume alcohol.

Conclusions. Risk perception of medicinal marijuana is 4.22 in medical students of northeast of Mexico. Students may underestimate its adverse effects. More studies with respect to this are needed.

Keywords

Medicinal marijuana, medical students, Mexico, Risk perception, Drugs

$\begin{array}{lll}\text { Open Peer Review } & \\ \text { Approval Status } & \mathrm{X} \\ & 1 & 2 \\ \text { version 1 } & \times & ? \\ 04 \text { Oct 2017 } & \text { view } & \text { view }\end{array}$

1. Nicholas Chadi (D), Boston Children's Hospital, Boston, USA

Sharon Levy, Boston Children's Hospital, Boston, USA

2. Sultan Alnohair, Qassim University,

Buraydah, Saudi Arabia

Any reports and responses or comments on the article can be found at the end of the article. 
Corresponding author: Omar González-Santiago (omargs28@yahoo.com)

Author roles: Castillo-Guzmán S: Conceptualization, Writing - Review \& Editing; Palacios-Ríos D: Formal Analysis, Methodology; NavaObregón TA: Formal Analysis, Methodology; Arredondo-Mendoza JC: Data Curation, Investigation; Alcalá-Alvarado OV: Investigation, Writing - Original Draft Preparation; Alonso-Bracho SA: Investigation, Writing - Original Draft Preparation; Becerril-Gaitán DA: Investigation, Writing - Original Draft Preparation; González-Santiago O: Conceptualization, Supervision, Writing - Review \& Editing

Competing interests: No competing interests were disclosed.

Grant information: The author(s) declared that no grants were involved in supporting this work.

Copyright: @ 2017 Castillo-Guzmán S et al. This is an open access article distributed under the terms of the Creative Commons Attribution License, which permits unrestricted use, distribution, and reproduction in any medium, provided the original work is properly cited. Data associated with the article are available under the terms of the Creative Commons Zero "No rights reserved" data waiver (CC0 1.0 Public domain dedication).

How to cite this article: Castillo-Guzmán S, Palacios-Ríos D, Nava-Obregón TA et al. Risk perception of medicinal marijuana in medical students from northeast Mexico [version 1; peer review: 1 approved with reservations, 1 not approved] F1000Research 2017, 6:1802 https://doi.org/10.12688/f1000research.12638.1

First published: 04 Oct 2017, 6:1802 https://doi.org/10.12688/f1000research.12638.1 


\section{Introduction}

Several studies have showed the potential benefits of medicinal marijuana (MM) for the treatment of some illnesses, such as pain in cancer, multiple sclerosis, Alzheimer's disease, post-traumatic stress disorder, epilepsy, Crohn's disease, and glaucoma ${ }^{1,2}$. However, like other drugs, MM is not exempt from serious adverse effects, such as psychiatric disorders, euphoria, disorientation, confusion, somnolence, and fatigue; therefore, it should be used with extreme caution $^{3}$. Legally, physicians cannot prescribe MM; however, in some countries, they can recommend its use when they consider it necessary. Some recommendations on how to prescribe marijuana for the care of patients have been published previously ${ }^{4}$.

On the other hand, risk perceptions towards medicinal drugs have been reported that influence in the prescribing behavior and the willingness of physicians to report adverse drug reactions. Knowing the risk perceived by medical students to drug prescription is important, since it establishes the requirement of education regarding certain medicines, like MM. In this respect, there are no studies regarding the risk perception toward MM in the general population and among health professionals. The existing literature has focused mainly on attitudes and support toward its legalization. Across countries, the factors associated with support for its legalization are political tolerance, ideology, and the views toward government ${ }^{5}$.

In Mexico, as in others countries, the use of MM was prohibited; however, in December 2016, the Senate approved the legalization of MM and sent the bill to the Chamber of Representatives for ratification, which occurred on April 28, 2017. The next steps for its appropriate use include the publication of laws, regulations, and guidelines by the Health Ministry, medical schools, and medical associations. While this continues, it is important that health professionals be updated on this topic, so that they can rationally recommend the use of MM. To achieve this, a first approach would be to know the attitudes and willingness to recommend the use of MM, and the risk perceived by physicians. In this study, we evaluate the risk perception of MM in medical students from northeast Mexico and determine associated factors.

\section{Methods}

\section{Study design}

A cross-sectional study that uses a visual scale to estimate risk perception of MM. The survey was applied during July and August 2017 in a public university from northeast of Mexico.

\section{Participants}

The inclusion criteria were as follow: Medical students of Universidad Autónoma de Nuevo León, both genders, any semester of study, and ages older than 18 years. Participants with incomplete surveys of second section were excluded. The medical students were contacted personally in the study areas and halls of the Faculty of Medicine. After obtaining verbal consent the survey was applied. This was self-administered with supervision. This study was performed in accordance with the Declaration of Helsinki and was approved by the Ethics Committee of the Faculty of Medicine at Universidad Autónoma de Nuevo León (registration number PI17-00134). Only verbal consent was requested from participants because the study is of low risk. This type of consent was approved by the Ethics Committee.

\section{Instrument}

The survey used was composed of two sections, the first section, which was optional, collected demographic information, such as age, gender, semester of study, self-reported alcohol and smoking status (undefined level, only yes or no), and currently selfreported disease (unspecific, only yes or no). The second section evaluates the risk perception toward the use of MM. For this, a visual scale of $10 \mathrm{~cm}$, from $0 \mathrm{~cm}$ (without risk) to $10 \mathrm{~cm}$ (completely risky), was used. Participants marked over the scale the risk that they perceived when MM is used. This section also contained two additional scales for paracetamol and sedatives, which act as relatively safe (negative control) and risky (positive control) drugs, respectively. The order of scales, including MM, was randomly allocated. The use of this visual $10-\mathrm{cm}$ scales has been used previously in studies that assess the risk perception to other medicinal drugs $\mathrm{s}^{6-8}$. The complete survey is available as Supplementary File 1.

\section{Statistical analysis}

Descriptive statistics is reported for demographic data. Risk perception was reported as the median of centimeters marked over the scale (from 0 to the mark). The results of risk perception were grouped by three age groups (18-20, 21-23 and >23 years), gender, semester of study (1-3, 4-7 and >7 semester), and selfreported alcohol and smoking status. Differences among groups were evaluated with the Mann-Whitney and Kruskal-Wallis tests, as appropriate. The statistical package NCSS version 11 was used for all analysis. The level of significance was $\mathrm{p}<0.05$.

\section{Results}

Overall, the rate of response was $97 \%$. In total 283 students were interviewed, of which $50.8 \%$ were men; $61 \%$ had an age range of 21-23 years, and $62.8 \%$ were enrolled in semesters 8 or higher. In addition, $18.7 \%$ self-reported smoking, $48.1 \%$ consuming alcohol and $14.1 \%$ self-reported having a (unspecified) disease (Table 1).

Overall, the risk perception of MM was $4.22 \mathrm{~cm}$, while for paracetamol and sedatives it was 1.56 and $5.00 \mathrm{~cm}$, respectively (Figure 1). The observed differences among the three drugs were statistically significant $(\mathrm{P}<0.05)$. This pattern was similar according to gender, age, semester of study, smoking and alcohol consumption and having some (unspecified) disease.

The analysis of individual drugs did not show a significant difference between gender, smoking and alcoholism status, having a disease, or among age groups and the semester of study in the risk perception of paracetamol and sedatives (Table 2). However, a significant difference was observed between self-reported smoking and alcohol consumption in the risk perception of MM. Those that self-reported smoking or alcoholism had a lower risk perception of MM. 
Table 1. Sociodemographic characteristics of participants.

\begin{tabular}{|c|c|c|}
\hline Variable & $\mathbf{N}$ & $\%$ \\
\hline Total & 283 & 100 \\
\hline \multicolumn{3}{|l|}{ Gender } \\
\hline Male & 144 & 50.8 \\
\hline Female & 139 & 49.1 \\
\hline \multicolumn{3}{|l|}{$\mathrm{Age}^{*}$} \\
\hline $18-20$ & 71 & 25.6 \\
\hline $21-23$ & 169 & 61.0 \\
\hline$>24$ & 37 & 13.4 \\
\hline \multicolumn{3}{|c|}{ Semester of study } \\
\hline $1-3$ & 49 & 17.4 \\
\hline $4-7$ & 56 & 19.9 \\
\hline$>8$ & 177 & 62.8 \\
\hline \multicolumn{3}{|l|}{ Smoking } \\
\hline Yes & 53 & 18.7 \\
\hline No & 230 & 81.3 \\
\hline \multicolumn{3}{|l|}{ Alcohol } \\
\hline Yes & 136 & 48.1 \\
\hline No & 147 & 51.9 \\
\hline \multicolumn{3}{|c|}{ Have a disease } \\
\hline Yes & 40 & 14.1 \\
\hline No & 243 & 85.9 \\
\hline
\end{tabular}

Table 2. Risk perception of medicinal marijuana (MM) by sociodemographic characteristics in medical students, using a visual scale with the median of $\mathrm{cm}$.

\begin{tabular}{|c|c|c|c|c|}
\hline Variable & MM & Paracetamol & Sedatives & P value \\
\hline Total & 4.22 & 1.56 & 5.00 & $<0.01$ \\
\hline \multicolumn{5}{|l|}{ Gender } \\
\hline Male & 3.95 & 1.22 & 4.89 & $<0.01$ \\
\hline Female & 4.44 & 1.44 & 5.00 & $<0.01$ \\
\hline$P$ value & 0.17 & 0.83 & 0.66 & \\
\hline \multicolumn{5}{|l|}{ Age } \\
\hline $18-20$ & 4.22 & 1.33 & 5.22 & $<0.01$ \\
\hline $21-23$ & 4.22 & 1.39 & 4.89 & $<0.01$ \\
\hline$>24$ & 4.00 & 1.56 & 4.89 & $<0.01$ \\
\hline$P$ value & 0.64 & 0.89 & 0.28 & \\
\hline \multicolumn{5}{|c|}{ Semester of study } \\
\hline $1-3$ & 3.00 & 1.22 & 4.84 & $<0.01$ \\
\hline $4-7$ & 4.67 & 1.22 & 4.78 & $<0.01$ \\
\hline$>8$ & 4.22 & 1.56 & 5.00 & $<0.01$ \\
\hline$P$ value & 0.19 & 0.36 & 0.69 & \\
\hline \multicolumn{5}{|l|}{ Smoking } \\
\hline Yes & 2.78 & 1.22 & 4.89 & $<0.01$ \\
\hline No & 4.39 & 1.44 & 5.00 & $<0.01$ \\
\hline$P$ value & 0.02 & 0.28 & 0.91 & \\
\hline \multicolumn{5}{|l|}{ Alcohol } \\
\hline Yes & 3.67 & 1.22 & 5.00 & $<0.01$ \\
\hline No & 4.56 & 1.56 & 4.89 & $<0.01$ \\
\hline$P$ value & 0.03 & 0.16 & 0.66 & \\
\hline \multicolumn{5}{|c|}{ Have a disease } \\
\hline Yes & 4.11 & 2.56 & 4.78 & $<0.01$ \\
\hline No & 4.22 & 1.33 & 5.00 & $<0.01$ \\
\hline$P$ value & 0.71 & 0.15 & 0.71 & \\
\hline
\end{tabular}

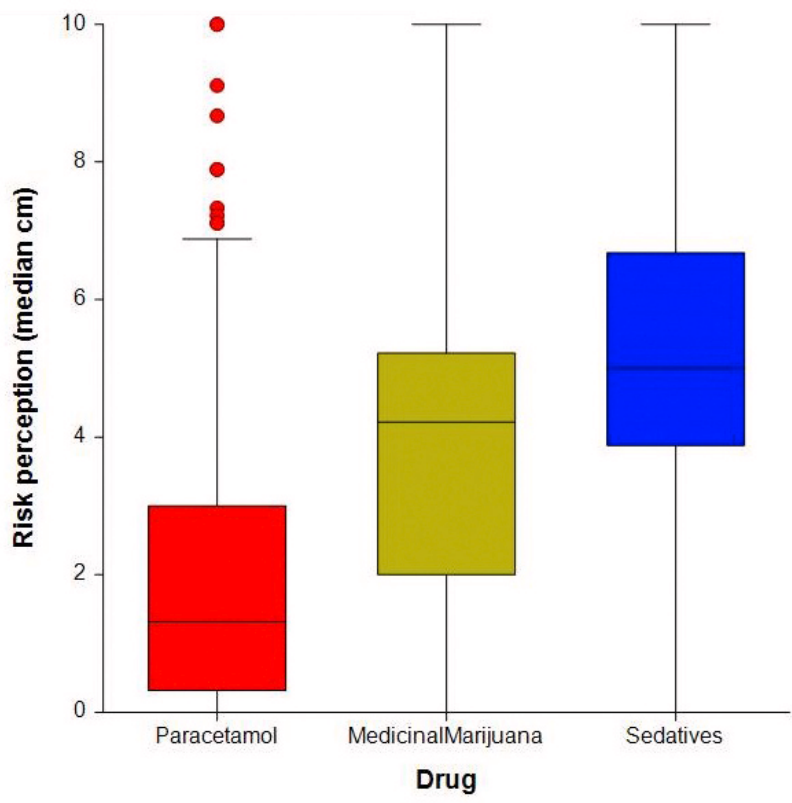

Figure 1. Risk perception of medicinal marijuana among medical students in northeast of Mexico. Two additional scales for paracetamol and sedatives are included, which act as relatively safe (negative control) and risky (positive control) drugs. 
Dataset 1. Raw data of risk perception to medicinal marijuana in medical students

http://dx.doi.org/10.5256/f1000research.12638.d179069

\section{Discussion}

In this study, we assessed the risk perceived by medical students toward the use of MM. Previous reports have analyzed the risk perception to several drug prescriptions; however, this is the first study that analyzes the risk perception to MM using the same instrument that these studies used.

Although several studies in the general population and among health professionals have shown a support for the legal use of $\mathrm{MM}^{9-12}$, physicians undoubtedly require solid knowledge of both its benefits and adverse effects if they want recommend it appropriately. Our results show that the risk perceived to MM $(4.2 \mathrm{~cm})$ is higher than paracetamol but lower than sedatives in Mexican medical students. Compared with other studies, the risk perceived is similar to the risk perceived to antibiotics, hypocholesterolemia drugs, and antihypertensives (median range 3-5 cm), but lower than that to nonsteroidal anti-inflammatory drugs (NSAIDs), antidepressants, and anticoagulants (median $>6 \mathrm{~cm})^{6-8,13}$. Although the objective was not to establish whether perceived risk was adequate, we speculate that observed outcome is low. We think that a reasonable risk perception of MM should be more than 6 , close to other drugs as sedatives, antidepressants or anticoagulants. This due to the difficulty of dosing, specifically if it is smoked, and the frequency of adverse effects of MM. We speculate that students with risk perception values of MM lower than 5 could underestimate its adverse effects and probably could recommend it indiscriminately during their practice. More studies concerning this are needed.

On the other hand, the risk perception of paracetamol was low and is similar to that previously reported ${ }^{14}$. The implications of this finding could be the same as with MM; students could underestimate its adverse effects, which is mainly liver damage ${ }^{15}$. It is worth remembering that lack of awareness of potential harm from taking or administering paracetamol improperly in adults and adolescents is a cause of emergency department visits ${ }^{16}$.

An interesting result was the significant difference in risk perception between those that self-reported smoking and alcohol consumption. The users of these recreational drugs had a lower risk perception of MM. In this sense, it has been proven in previous studies that tobacco and alcohol consumption are risk factors for the use of recreational marijuana ${ }^{17,18}$. In addition, the consumption of these drugs has been associated with support for legalization of recreational and $\mathrm{MM}^{19,20}$.

The risk perception observed could be due in part to attention from mass media regarding its potential uses. Previous studies have found an association between public support for MM and its coverage in media ${ }^{21,22}$. Another factor that could impact risk perception values, is the lack of formal courses regarding MM in the syllabus of students surveyed ${ }^{23}$. In this sense, previous studies have proven that formal courses of pharmacology increase risk perception toward common drugs like NSAIDs ${ }^{7}$. The design and implementation of formal courses regarding MM may have the same impact.

Finally, we consider that our results should be interpreted with caution, as it is possible that our findings may not be generalized to other countries, due to differences in teaching methods. Replication in others countries, especially where the use of MM has been recently made legal, is needed. Although the risk perception of drugs has been studied with visual scales, the development of other instruments could improve the assessment.

\section{Conclusions}

The risk perception of MM was 4.22 in medical students of the northeast of Mexico. With the legalization of MM in Mexico, formal courses regarding dosing, and adverse and beneficial effects of MM will be needed in medical schools.

\section{Ethical statement}

This study was performed in accordance with the Declaration of Helsinki and was approved by the Ethics Committee of the Faculty of Medicine (registration number, PI17-00134).

\section{Data availability}

Dataset 1: Raw data of risk perception to medicinal marijuana in medical students. doi, 10.5256/f1000research.12638.d179069²4

\section{Competing interests}

No competing interests were disclosed.

\section{Grant information}

The author(s) declared that no grants were involved in supporting this work.

\section{Acknowledgements}

To all medical students that participated in this study. To Dr Sergio Lozano for his help in the edition of manuscript.

\section{Supplementary material}

Supplementary File 1: Survey in English and Spanish.

Click here to access the data. 
1. Whiting PF, Wolff RF, Deshpande S, et al:: Cannabinoids for Medical Use: A Systematic Review and Meta-analysis. JAMA. 2015; 313(24): 2456-2473. PubMed Abstract | Publisher Full Text

2. Wilkie G, Sakr B, Rizack T: Medical Marijuana Use in Oncology: A Review. JAMA Oncol. 2016; 2(5): 670-675.

PubMed Abstract | Publisher Full Text

3. Ciccone CD: Medical Marijuana: Just the Beginning of a Long, Strange Trip? Phys Ther. 2017; 97(2): 239-248.
PubMed Abstract | Publisher Full Text

4. Chaudhry HJ, Hengerer AS, Snyder GB: Medical Board Expectations for Physicians Recommending Marijuana. JAMA. 2016; 316(6): 577-578. PubMed Abstract | Publisher Full Text

5. Cruz JM, Queirolo R, Boidi MF: Determinants of public support for marijuana legalization in uruguay, the united states, and el salvador. J Drug Issues. 2016 46(4): 308-325.

Publisher Full Text

6. Bongard V, Ménard-Taché S, Bagheri $\mathrm{H}$, et al.: Perception of the risk of adverse drug reactions: differences between health professionals and non health professionals. Br J Clin Pharmacol. 2002; 54(4): 433-436. PubMed Abstract | Publisher Full Text | Free Full Text

7. Durrieu G, Hurault C, Bongard V, et al.: Perception of risk of adverse drug reactions by medical students: influence of a 1 year pharmacological course. Br J Clin Pharmacol. 2007; 64(2): 233-236. PubMed Abstract | Publisher Full Text | Free Full Text

8. Buendia JA, Zuluaga AF: [Physicians' insight about adverse drug reaction to frequently used medication groups in Bogotá (Colombia)]. Biomedica. 2014; 34(3): 403-8.

PubMed Abstract

9. Support increases for marijuana legalization | Pew Research Center [Internet] [cited 2017 May 5]

Reference Source

10. Millhorn M, Monaghan M, Montero D, et al:: North americans' attitudes toward illegal drugs. J Hum Behav Soc Environ. 2009; 19(2): 125-141. Publisher Full Text

11. Chan MH, Knoepke CE, Cole ML, et al.: Colorado Medical Students' Attitudes and Beliefs About Marijuana. J Gen Intern Med. 2017; 32(4): 458-463. PubMed Abstract | Publisher Full Text | Free Full Text

12. Kondrad E, Reid A: Colorado family physicians' attitudes toward medical marijuana. J Am Board Fam Med. 2013; 26(1): 52-60. PubMed Abstract | Publisher Full Text

13. Cullen G, Kelly E, Murray FE: Patients' knowledge of adverse reactions to current medications. Br J Clin Pharmacol. 2006; 62(2): 232-236. PubMed Abstract | Publisher Full Text | Free Full Text
14. Castillo-Guzman S, González-Santiago O, Delgado-Leal IA, et al:: Perception of the risk of adverse reactions to analgesics: differences between medica students and residents. PeerJ. 2016; 4: e2255. PubMed Abstract | Publisher Full Text | Free Full Text

15. Tittarelli R, Pellegrini M, Scarpellini MG, et al:: Hepatotoxicity of paracetamol and related fatalities. Eur Rev Med Pharmacol Sci. 2017; 21(1 Suppl): 95-101. PubMed Abstract

16. Budnitz DS, Lovegrove MC, Crosby AE: Emergency department visits fo overdoses of acetaminophen-containing products. Am J Prev Med. 2011; 40(6): 585-592.

PubMed Abstract | Publisher Full Text

17. von Sydow $\mathrm{K}$, Lieb R, Pfister $\mathrm{H}$, et al.: What predicts incident use of cannabis and progression to abuse and dependence? A 4-year prospective examination of risk factors in a community sample of adolescents and young adults. Drug Alcohol Depend. 2002; 68(1): 49-64. PubMed Abstract | Publisher Full Text

18. Iglesias V, Cavada G, Silva C, et al:: [Early tobacco and alcohol consumption as modifying risk factors on marijuana use]. Rev Saude Publica. 2007; 41(4) 517-522. PubMed Abstract | Publisher Full Text

19. Sznitman SR, Bretteville-Jensen AL: Public opinion and medical cannabis policies: examining the role of underlying beliefs and national medical cannabis policies. Harm Reduct J. 2015; 12: 46. PubMed Abstract | Publisher Full Text | Free Full Text

20. Van der Sar R, Brouwers EPM, van de Goor LAM, et al.: The opinion on Dutch cannabis policy measures: A cross-sectional survey. Drugs Education Prevention and Policy. 2011; 18(3): 161-171. Publisher Full Text

21. Sznitman SR, Lewis N: Is cannabis an illicit drug or a medicine? A quantitative framing analysis of Israeli newspaper coverage. Int J Drug Policy. 2015; 26(5): 446-452.

PubMed Abstract | Publisher Full Text

22. Santos MT, Camacho I: El tratamiento del cannabis en la prensa española. Cuadernos info. 2017; 153-171. Publisher Full Text

23. Programa Académico Universidad Autónoma de Nuevo León. Facultad de Medicina [Internet]. [cited 2017 Sep 10]. Reference Source

24. Castillo-Guzmán S, Palacios-Ríos D, Nava-Obregón TA, et al.: Dataset 1 in: Risk perception of medicinal marijuana in medical students from northeast Mexico. F1000Research. 2017.

Data Source 


\section{Open Peer Review}

\section{Current Peer Review Status: $\mathrm{X}$ ?}

\section{Version 1}

Reviewer Report 08 April 2019

https://doi.org/10.5256/f1000research.13684.r46028

(c) 2019 Alnohair S. This is an open access peer review report distributed under the terms of the Creative Commons Attribution License, which permits unrestricted use, distribution, and reproduction in any medium, provided the original work is properly cited.

\section{Sultan Alnohair}

Department of Family and Community Medicine, College of Medicine, Qassim University, Buraydah, Saudi Arabia

The discussion of relevant epidemiological data conducted in Mexico and other countries could be included. Correlates such as socio-demographic, culture-related factors of Mexico and other countries pertaining to adolescent smoking could also be discussed in the Introduction Section.

Methods - The information below is missing in the paper but should be included:

1. The authors did not tell us how many students participated in the study and how many of them was included and excluded?

2. The criteria for selection of the participating medical students should be listed.

3. What is the percentage of those who participated compared to the total medical students?

4. Is it daily interview or once per week from Jun up to August?

5. What were the inclusion/exclusion criteria of the participants?

6. Regarding the assessment, the authors need to let the readers know more about the variables which were included in the questionnaire. The authors should state the scales that have been used to measure the variables, the reliability etc. All of those were missing in the Methods Section.

7. Since the authors outlined some information about northeast Mexico, it would be better if the authors could devote a section to discuss northeast Mexico in the Introduction part so as to let the readers understand what are the existing cultural factors affecting adolescents in that area.

8. The authors also need to state that the rights and confidentiality of the participants have been communicated to them. I suggest to make correlation between smoking status and their perception.

9. Also, the survey should mention about the family history of smoking, using marijuana in the home.

10. The study was done on medical students but actually no clear question about the effect of medical schools on their perception. How many lectures per semester talk about drug 
abuse.

The study has implications on policy and how adolescents are educated to resist peer pressure when it comes to smoking.

In general, a good study but needs more clarification about the comments above and how the medical school will increase the awareness of medical students about the harmful of MM.

Is the work clearly and accurately presented and does it cite the current literature? Partly

Is the study design appropriate and is the work technically sound?

Partly

Are sufficient details of methods and analysis provided to allow replication by others? No

If applicable, is the statistical analysis and its interpretation appropriate?

Not applicable

Are all the source data underlying the results available to ensure full reproducibility? Yes

Are the conclusions drawn adequately supported by the results? No

Competing Interests: No competing interests were disclosed.

Reviewer Expertise: -chronic disease as Diabetes ,Hypertension

I confirm that I have read this submission and believe that I have an appropriate level of expertise to confirm that it is of an acceptable scientific standard, however I have significant reservations, as outlined above.

Reviewer Report 07 November 2017

https://doi.org/10.5256/f1000research.13684.r27228

(C) 2017 Levy S et al. This is an open access peer review report distributed under the terms of the Creative Commons Attribution License, which permits unrestricted use, distribution, and reproduction in any medium, provided the original work is properly cited.

Nicholas Chadi

Division of Developmental Medicine, Boston Children's Hospital, Boston, MA, USA

Sharon Levy

Division of Developmental Medicine, Boston Children's Hospital, Boston, MA, USA

Overarching comments: 
This article describes the results of a cross-sectional study that used a self-administered survey to explore the perception of riskiness of medical marijuana in comparison to paracetamol and sedatives among a sample of medical students in a single medical school in northeast Mexico. The study addresses an important and timely topic given the current movement towards legalization of medicinal marijuana in North America. Also, provider perception of risk associated with medicinal marijuana has not previously been reported.

However, in its current state, the manuscript is unfit for publication. The introduction is insufficiently supported by evidence, and contains several grammatical and structural mistakes that make it very difficult to read. The aims and objectives of the study are not clearly stated. The methodology has several gaps and generally lacks in rigor. Specifically, the question asked to study participants is unclear ("What is the level of risk associated with medicinal marijuana") as participants are not told what type of risk we are concerned about. The discussion section also has several grammatical mistakes, includes several statements that are not supported by evidence, and does not adequately explain the significance of the findings. Also, the authors fail to underline key limitations in their study. Finally, the conclusions are not appropriately related to study data and lack in clinical relevance.

All in all, the authors have attempted to research an important issue, but the level of rigor in their investigation is insufficient. The survey designed by the authors fails to ask a clear question to study participants and the very high number of grammatical mistakes in the manuscript severely impacts readability. We recognize the effort that was put into this study, and congratulate the authors for selecting an important and timely topic, but cannot recommend publication of this manuscript.

\section{Detailed comments:}

Consider changing the term medicinal marijuana to medical marijuana (this term is more commonly used in North America).

\section{Abstract:}

In the results section, the authors should clearly state that students who used alcohol or cigarettes had a lower risk perception of marijuana. Also, authors need to specify the units of the risk perception scale of put it into context.

The conclusions need to be rephrased. A score of 4.22 on a visual analogue scale does not have any clinical or practical significance in and of itself.

\section{Introduction:}

The text has several grammatical and syntactic mistakes that make it difficult to read.

Several statements in the Introduction are not supported by a reference. This needs to be addressed.

The second paragraph is very difficult to read and needs to be rewritten.

In the third paragraph, the authors appear to support the recommendation of medical marijuana for patients ("...so that they can rationally recommend the use of MM..."). Authors refrain from making recommendations based on personal opinion or rewrite this sentence so that it appears neutral.

The aims and objectives of the study are not clearly stated and should be clearly described. 


\section{Methods:}

The first sentence is grammatically incorrect and needs to be revised.

Participants: The reason for exclusion of participants with incomplete data is unclear (this data needs to be reported). The sentence where this is stated is ambiguous and grammatically incorrect.

Authors need to detail who obtained consent and who was providing supervision while participants were filling in their surveys as this could be a significant source of bias. Also, was privacy respected? This is a critical point.

Were surveys administered anonymously? This needs to be stated explicitly in the manuscript.

- It is unclear why the authors refer to the Declaration of Helsinki, this should be removed.

Instrument: It is unclear why the demographic section was optional. This highly impacts the results of the study.

It is unclear why the authors asked participants about having a disease.

Authors should say more explicitly how they asked the question on perceived riskiness of medical marijuana (this should appear in the methodology section).

\section{Results:}

In Table 1, authors need to be more specific about how much data is missing as this could introduce a source of bias

It is unclear what the response rate represents. Is it referring to the proportion of students who were offered to participate or is it the proportion of medical students at the medical school?

In Figure 1, authors state that paracetamol is safe and that sedatives are risky. This should be supported by evidence.

Table 2 would benefit from more detail in the row headers as they are hard to interpret. Also, authors should include interquartile ranges in Table 2. Finally, it is not clear what the p-values for the rows in Table 2 are comparing - highest to lowest values would mean comparing paracetamol to sedatives - presumably that is not what authors mean.

The term "control" is inadequate in this context.

While there is no statistically significant difference between semester of study, it looks like there could be a difference between students early in their studies (1-3) vs $>4$ if the data were dichotomized in this way. That could be interesting because it might suggest that as students learn more in school they become more aware of the risks of marijuana use.

\section{Discussion:}

The first sentence of the discussion should summarize the authors' research findings. Authors should acknowledge that there is a high level of controversy around the use of medical marijuana.

The authors compare the results of their study with results from other studies. This comparison needs to be detailed. Were all the studies asking the exact same question? The comment in the discussion that the "risk is too low and should be closer to 6" seems to have no justification, and should be removed (if the manuscript is to be edited). This is strictly the authors' speculation about a scale that has even been validated for this type of experiment (or has it? If it has been this needs to be mentioned in the manuscript) - in other words we don't even know what a 6/10 means, nor is there any evidence that the scale is 
anything more than ordinal. If the authors have more data about the scale and its validity they should include it.

The authors fail to recognize several methodological limitations in their study (ie: missing data, mode of recruitment, one single medical school...).

The authors' explanation of why the fact that students who use alcohol or cigarettes have lower risk perception is an interesting finding is incoherent.

The authors appropriately recognize opportunities for education but could make better use of study data to support their point. Ex: mention that the risk perception of MM does not change with the number of school semester, suggesting a potential missed opportunity in medical education, as students should become more aware of the risks of recommending MM if taught about those risks.

The authors appropriately recognized that the study findings might not be generalizable to other countries, but should also acknowledge that they have surveyed students and not healthcare providers.

In general, the discussion lacks in depth and clinical relevance.

Finally there is no sense of the relevance of this data. Are higher risk perceptions associated with less frequent recommendations for other substances or are they associated with some other meaningful impact on provider behavior? Without this, the data is decontextualized

Conclusions: and not easy to meaningfully interpret.

Conclusions lack clinical relevance and are not appropriately supported by the research data.

Is the work clearly and accurately presented and does it cite the current literature? No

Is the study design appropriate and is the work technically sound?

Partly

Are sufficient details of methods and analysis provided to allow replication by others? Partly

If applicable, is the statistical analysis and its interpretation appropriate? Partly

Are all the source data underlying the results available to ensure full reproducibility? Partly

Are the conclusions drawn adequately supported by the results? No

Competing Interests: No competing interests were disclosed.

Reviewer Expertise: Addiction medicine

We confirm that we have read this submission and believe that we have an appropriate level of expertise to state that we do not consider it to be of an acceptable scientific standard, for 
reasons outlined above.

Author Response 07 Nov 2017

Omar González-Santiago, Universidad Autónoma de Nuevo León, San Nicolas de Los Garza, Mexico

Thank you very much to Dr Chadi and Dr Levy for your comments regarding our manuscript. All your comments are important for us. I would like to clarify some points.

Grammatic will be revised by an profesional translator.

METHODS

Participants: The reason for exclusion of participants with incomplete data is unclear (this data needs to be reported). The sentence where this is stated is ambiguous and grammatically incorrect.

Response. At this respect we consider to exclude from the analysis the responses of participants with incomplete surveys of the second section. This is due to that the section correspond to risk perception of MM. Fortunately, all participants completed the second section so we will delete this sentence.

Authors should say more explicitly how they asked the question on perceived riskiness of medical marijuana (this should appear in the methodology section).

Response. The specific question will be written in the method section. However, you can find it in the survey, which is provided as a supplementary file.

RESULTS

In Figure 1, authors state that paracetamol is safe and that sedatives are risky. This should be supported by evidence.

Response. In Figure 1 we do not affirm that paracetamol and sedative are completely safe and risky respectively. We state that two additional scales were included for paracetamol and sedatives which act "RELATIVELY" safe and risky control. We agree with the term control is inadequate in this context and will be removed.

While there is no statistically significant difference between semester of study, it looks like there could be a difference between students early in their studies (1-3) vs $>4$ if the data were dichotomized in this way.

Response. Thank you very much for your observations. We will consider dichotomies as you recommend.

\section{DISCUSSION}

The comment in the discussion that the "risk is too low and should be closer to 6" seems to have no justification, and should be removed (if the manuscript is to be edited). This is strictly the authors' speculation about a scale that has even been validated for this type of experiment (or has it? If it has been this needs to be mentioned in the manuscript) - in other words we don't even know what a 6/10 means, nor is there any evidence that the scale is anything more than ordinal. If the authors have more data about the scale and its validity they should include it 
Response. Thank you very much, we have clearly written in the paragraph that "we speculate" at this respect and expose our reasons. In addition, we recommend more studies at this respect in the last sentence.

Finally there is no sense of the relevance of this data. Are higher risk perceptions associated with less frequent recommendations for other substances or are they associated with some other meaningful impact on provider behavior? Without this, the data is decontextualized and not easy to meaningfully interpret.

Response. With respect to MM there is no data. However, we comment the association of risk perception of medicinal drugs with behaviors of physicians in the second paragraph of introduction (it will be more referenced). These paragraph will be placed in discussion section for improve the sense of relevance.

Competing Interests: I declare that I do not have conflicts of interest

The benefits of publishing with F1000Research:

- Your article is published within days, with no editorial bias

- You can publish traditional articles, null/negative results, case reports, data notes and more

- The peer review process is transparent and collaborative

- Your article is indexed in PubMed after passing peer review

- Dedicated customer support at every stage

For pre-submission enquiries, contact research@f1000.com 